\title{
GT2014-25689
}

\section{COMPRESSIBLE DIRECT NUMERICAL SIMULATION OF LOW-PRESSURE TURBINES: PART II - EFFECT OF INFLOW DISTURBANCES}

\author{
Vittorio Michelassi \\ General Electric Global Research \\ Freisinger Landstrasse 50 \\ D-85748, Garching b. München, Deutschland
}

\author{
Li-Wei Chen, Richard Pichler, Richard D. Sandberg* \\ Aerodynamics and Flight Mechanics Research Group \\ Faculty of Engineering and the Environment \\ University of Southampton \\ Southampton, SO17 1BJ, U.K. \\ Email: R.D.Sandberg@soton.ac.uk
}

\begin{abstract}
In the present paper, direct numerical simulation (DNS) studies of the compressible flow in the T106 linear cascade have been carried out. Various environmental variables, i.e. background turbulence level, frequency of incoming wakes and Reynolds number, and a combination of these were considered for a total of 12 fully resolved simulations. The mechanism$s$ dictating the observed flow phenomena, including the mixing and distortion of the incoming wakes, wake/boundary layer interaction, and boundary layer evolution impact on profile loss generation are studied systematically. A detailed loss generation analysis allows the identification of each source of loss in boundary layers and flow core. Particular attention is devoted to the concerted impact of wakes distortion mechanics and the intermittent nature of the unsteady boundary layer. Further, the present study examines the validity of the Boussinesq eddy viscosity assumption, which invokes a linear stress-strain relationship in commonly used RANS models. The errors originating from this assumption are scrutinized with both time and phaselocked averaged flow fields to possibly identify shortcomings of traditional RANS models.
\end{abstract}

\section{NOMENCLATURE}

$C$ blade chord length

$C_{a x} \quad$ blade axial chord length

$C_{p} \quad$ static pressure coefficient

$F_{\text {red }}$ reduced frequency of bars

$l_{b} \quad$ length of separation bubble

Ma Mach number

Re Reynolds number

\footnotetext{
*Address all correspondence to this author.
}

\author{
blade surface length \\ pitch of the blade \\ strain rate tensor \\ time \\ bar passing period \\ background turbulence level \\ reference velocity \\ velocity \\ momentum thickness \\ density \\ wall shear stress \\ bar passing phase \\ wake loss coefficient \\ wake loss profile
}

Sub and Superscripts

$\begin{array}{ll}1 & \text { inlet plane } \\ 2 & \text { exit plane } \\ \infty & \text { reference value } \\ i s & \text { isentropic } \\ t & \text { stagnation quantity } \\ w & \text { quantity on the surface } \\ A & \text { mass flow averaged value } \\ M & \text { mixed-out quantity }\end{array}$

\section{INTRODUCTION}

In recent years, the interest in aircraft engine low pressure turbines (LPT) has considerably increased. The motivation is two-fold: a) the continuous growth of turbofan by-pass ratio to improve propulsion efficiency, and b) the observation that onepoint of LPT efficiency is worth 0.6-0.8 points of specific-fuelconsumption. Unfortunately, the larger the by-pass ratio the lower the RPM of the fan in order to control the fan tip relative Mach number. For direct drive engines this reflects in highly loaded 
LPT, while for geared fans the LPT load does decrease. Both engines type require a careful unsteady aerodynamic design in a wide Reynolds number ( $\mathrm{Re}$ ) range, from $30 \mathrm{~K}$ for a narrow-body aircraft at cruise, up to $200 \mathrm{k}-300 \mathrm{~K}$ for a wide-body at take-off.

Prakash et al. [1] reported an extensive experimental study on LPT profile losses by varying the midspan Zweifel number and several other design parameters for $\mathrm{Re}$ in the $70 \mathrm{k}$ to $350 \mathrm{k}$ range. Results showed risk of suction as well as pressure side separation. One of the first attempts to predict the unsteady flow in an LPT is by Arndt [2] who described the unsteady flow by measurements in the axial blade-row gaps. Halstead at al. [3] investigated a similar type of flow in a low speed multi-stage LP$\mathrm{T}$ facility, measuring accurate time averaged and instantaneous quantities. With an eye on unsteady effects, Coull et al. [4] studied the performance of LPT profiles and quantified the complex interaction between blade load (front-aft) and optimal diffusion factor under different Reynolds number and reduced frequency. They concluded that optimum performance could be obtained by carefully balancing the position of peak suction and diffusion ratio with incoming wakes reduced frequency.

Profile losses are considered the major source of inefficiency (above 60\%) in LPT. Stadtmüeller and Fottner [5,6] produced a detailed data set for profile loss model validation by measuring the flow in a LPT cascade without and with incoming wakes at different load levels. Their data-set was used by many authors to benchmark CFD, like Michelassi et al. [7], who compared DNS, LES, and URANS predictions. Although URANS, still the workhorse in design, were improved by comparing with DNS and LES, they still have difficulties in predicting profile losses due to boundary layer transition and/or separation. Also Medic and Sharma [8], who used proprietary profiles, had to switch to LES to predict the trend in losses with blade load style without incoming wakes. Simple two-equation turbulence models are unable to predict transition [7], although recent transition models, like those by Langtry and Menter [9], Keadle and McQuilling [10], or Walters and Cokljat [11] improve predictions. Nevertheless, there is still uncertainty on the contribution to losses of suction and pressure side separation and wakes.

To clarify the unsteady fluid dynamics in LPTs, Wu et al. [12] performed the DNS at $\mathrm{Re}=60 \mathrm{k}$ of the T106 airfoil with incoming wakes. Their analysis showed a complex turbulence production mechanism, that simple eddy viscosity models are not able to capture. Michelassi et al. [13] performed an LES of the same blade at $\mathrm{Re}=148 \mathrm{k}$, observing noticeable differences between DNS and LES for transition. This latter analysis confirmed that LES can have difficulties in predicting unsteady transition, as also highlighted by Durbin and Wu [14]. Hence, properly resolved and validated DNS, supported by experiments under controlled conditions, appears to be the best way to investigate all the complex mechanisms highlighted before and identify weaknesses in turbulence models routinely used in design.

Most of the DNS and LES mentioned above refer to a limited number of cases, and focus on flow details. The DNS subject of this paper focuses on the T106 cascade [6] and provides a comparative study of several operating conditions to shed light not only on flow patterns, but also on the loss generation mechanism.

\section{COMPUTATIONAL OVERVIEW}

The compressible Navier-Stokes equations for the conservative variables are solved using the DNS code, HiPSTAR [15]. The numerical method comprises a five-step, fourth-order accurate low-storage Runge-Kutta method for the time integration, state-of-the-art parallelizable wavenumber optimized compact finite differences for the spatial discretization in the streamwise and pitchwise directions, and a Fourier method using the FFTW3 library for discretization of the spanwise direction. Additionally, a skew-symmetric splitting is used to stabilize the convective terms. More details about the numerical method, mesh resolution and domain size can be found in part I of this paper.

The linear turbine cascade geometry in the present work is the T106 profile experimentally investigated by Stadtmüller [6]. At the outlet boundary a non-reflective characteristic boundary condition was applied. At the inlet boundary, a fixed inflow condition with an inflow angle of $\alpha=46.1^{\circ}$ was specified and a sponge layer, forcing the flow solution to a target state, was employed in the inlet region to remove unphysical acoustic waves [16]. To generate periodically incoming bar wakes, an immersed boundary method is implemented using feedback forcing terms proposed by Goldstein et al. [17]. In the numerical setup with incoming wakes, as shown in Fig. 1, each cylinder bar at $x / C=-0.7$ with a diameter of $0.02 C$ contains 90 time-varying immersed boundary points and the velocity in the $y$-direction is $V_{b a r}=-0.41 U_{\infty}$. Through changing the bar spacing, various reduced frequencies $F_{\text {red }}=f \cdot C / V_{2 i s}$ can be achieved, where $f$ and $V_{2 i s}$ are bar passing frequency and isentropic exit velocity, respectively. The cascade inlet measurement plane 1 is at $30 \%$ chord upstream of the blade leading edge and the exit measurement plane 2 at $40 \%$ chord downstream of the trailing edge.

The present study is aimed at investigating the effect of incoming bar wakes at different reduced frequencies and with and without background turbulence. As summarized in table 1 , twelve typical cases at isentropic Reynolds numbers $R e_{2 i s}=$ 60,000 and $100,000, F_{\text {red }}=0-1.22$ and $T u=0.5 \%, 1.2 \%$ and $3.2 \%$ are carried out where the isentropic Mach number $M_{2 i s}=0.405$ is kept the same for all cases. For each case, the simulation was run for 5 pass through times in 2D, then $3 \mathrm{D}$ simulations were restarted from the fully developed 2D result. After about 10 pass through times, to avoid the transient period, samples were collected for 20 bar passing periods to obtain statistically meaningful turbulence properties.

\section{RESULTS AND DISCUSSION Instantaneous Flow Structures}

For a qualitative view of coherent structures in the flow field, Fig. 2 shows two typical snapshots of flow field $\left(F_{\text {red }}=0.31\right.$ with $T u=0.5 \%$ at $\left.R e_{2 i s}=60,000\right)$ depicted by isosurfaces of the $Q$ criterion [18]. A positive value of $Q$ presents the regions in which the rotation exceeds the strain. Looking at Fig. 2(a), the cylinder wakes develop downstream into highly three dimensional structures and interact with the blade boundary layers. Due to the strongly strained flow in the vicinity of the leading edge, the eddies from the incoming wake are highly stretched near the leading edge, as a result of which the local streamwise vorticity is enhanced significantly. The incoming wakes go into the blade 


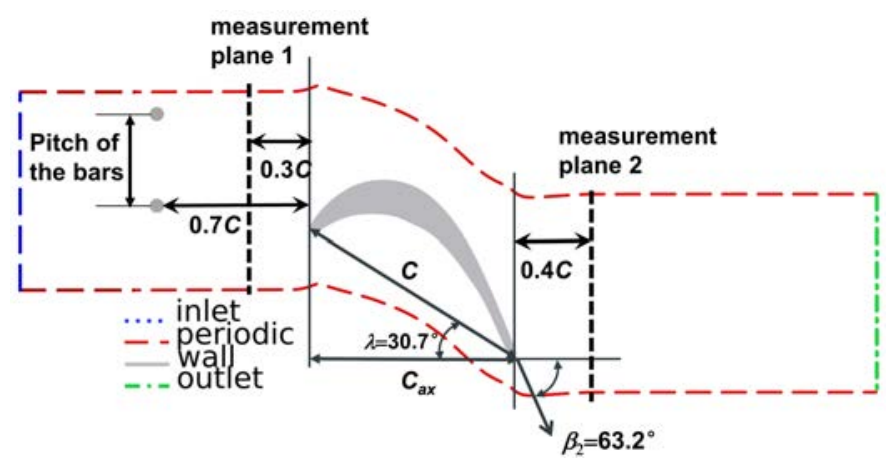

FIGURE 1: SKETCH OF MOVING BARS AND THE BLADE GEOMETRY.

TABLE 1: SUMMARY OF CASES

\begin{tabular}{ccc}
\hline \hline $\operatorname{Re}_{2 i s}$ & $F_{\text {red }}$ & $T u$ \\
\hline $60 \mathrm{k}$ & 0 & $0.5 \%, 1.2 \%, 3.2 \%$ \\
& 0.31 & $0.5 \%, 3.2 \%$ \\
& 0.61 & $0.5 \%, 3.2 \%$ \\
& 1.22 & $0.5 \%$ \\
\hline $100 \mathrm{k}$ & 0 & $0.5 \%, 3.2 \%$ \\
& 0.61 & $0.5 \%$ \\
& 1.22 & $0.5 \%$ \\
\hline \hline
\end{tabular}

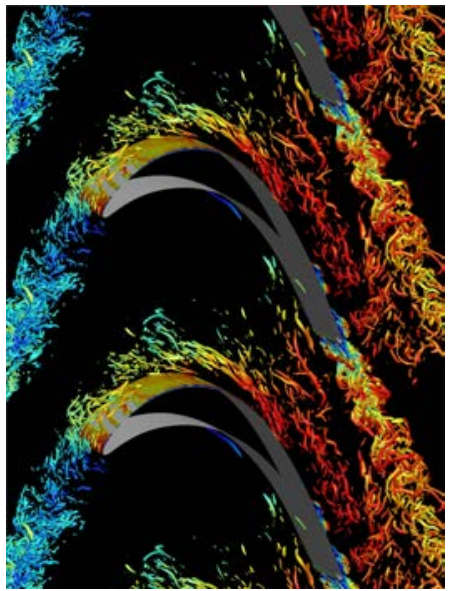

(a)

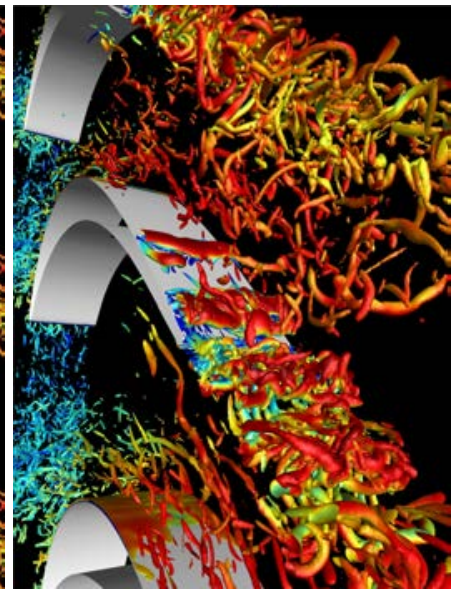

(b)
FIGURE 2: ISO-SURFACES OF INSTANTANEOUS SECOND INVARIANT OF THE VELOCITY-GRADIENT TENSOR $(Q=500)$ COLORED BY VELOCITY MAGNITUDE AT VARIOUS TIME INSTANTS FOR $F_{\text {red }}=0.31, T u=0.5 \%$, $R e_{2 i s}=60,000$.

passage, distort and then eventually merge with the blade wake. Understanding the evolution of the boundary layer and separation bubble on the suction side is of particular interest. As shown in Fig. 2(b), due to the Kelvin-Helmholtz instability the sepa-

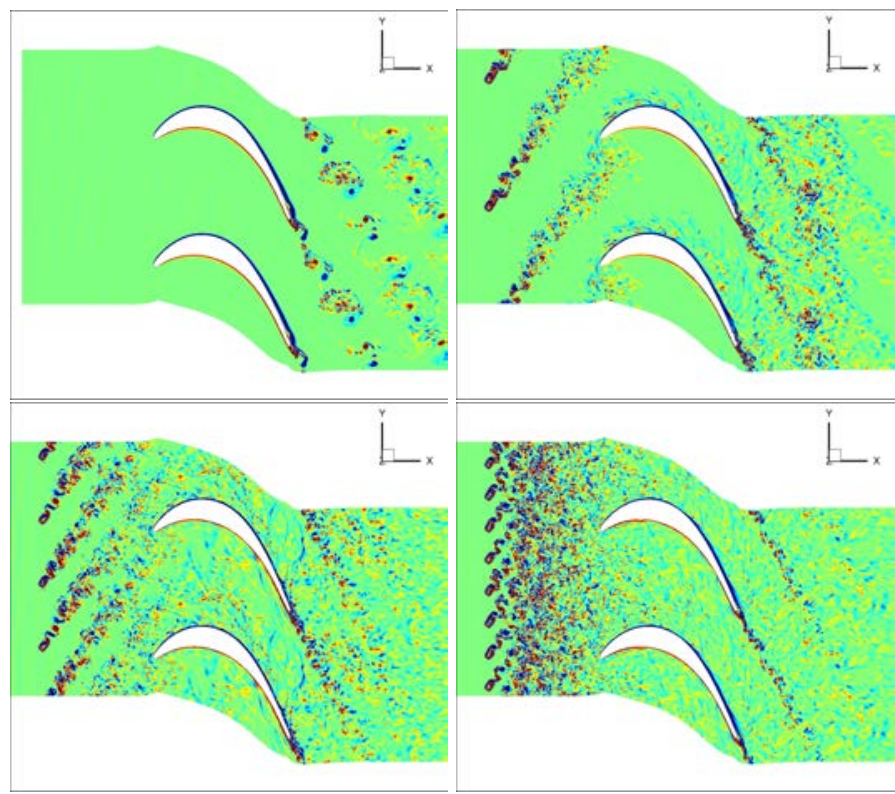

FIGURE 3: INSTANTANEOUS CONTOURS OF THE SPANWISE COMPONENT OF VORTICITY (-50 TO 50) IN THE MID-SPAN $(x, y)$ PLANE FOR $F_{r e d}=0$ (TOP LEFT), $F_{r e d}=$ 0.31 (TOP RIGHT), $F_{r e d}=0.61\left(\right.$ BOTTOM LEFT) AND $F_{r e d}=$ 1.22 (BOTTOM RIGHT) WITH $T u=0.5 \%, R e_{2 i s}=60,000$.

rated shear layer rolls up and breaks down into complex threedimensional structures further downstream. Influenced by the incoming wakes, the vortex-tube like structures shrink and disappear periodically, indicating that the separation bubble is suppressed.

Fig. 3 shows the instantaneous contours of the spanwise vorticity component in the mid-span $(x, y)$ plane for varying reduced frequencies at $R e_{2 i s}=60,000$ with $T u=0.5 \%$. In the case of $F_{\text {red }}=0$, as laminar flow separation occurs in the aft section of the suction side, highly unsteady vortex shedding can be observed. The blade wake developing downstream of the trailing edge shows very organized mushroom-like structures in the $F_{\text {red }}=0$ case. Once adding moving bars in the upstream of the blade passage, in the $F_{\text {red }}=0.31$ and $F_{\text {red }}=0.61$ cases, the bar wakes generated by moving cylinders develop downstream into highly 3D structures and interact with the blade boundary layers. For the $F_{\text {red }}=1.22$ case, the interaction between the bar wakes becomes strong. The incoming wake flow structures look similar to those of the turbulent inflow case without wakes. Compared to the case of $F_{\text {red }}=0$, in these moving bar cases, the blade wakes show less-organized structures and the vortex breakdown can be clearly noticed.

\section{Mean Flow Features}

For a more quantitative assessment of the effect of reduced frequency, background turbulence and Reynolds number on the blade performance, the pressure coefficient $C_{p}=\left(p_{w}-\right.$ $\left.p_{2}^{A}\right) /\left(p_{t, 1}^{A}-p_{2}^{A}\right)$ and the wall shear $\tau_{w}$ distributions for all cases are compared. Here $A$ denotes a mass flow averaged quantity. As shown in Fig. 4(a), the result of the $F_{\text {red }}=0$ and $F_{\text {red }}=0.61$ cases with $T u=0.5 \%$ (clean case) at $R e_{2 i s}=60,000$ compare well 

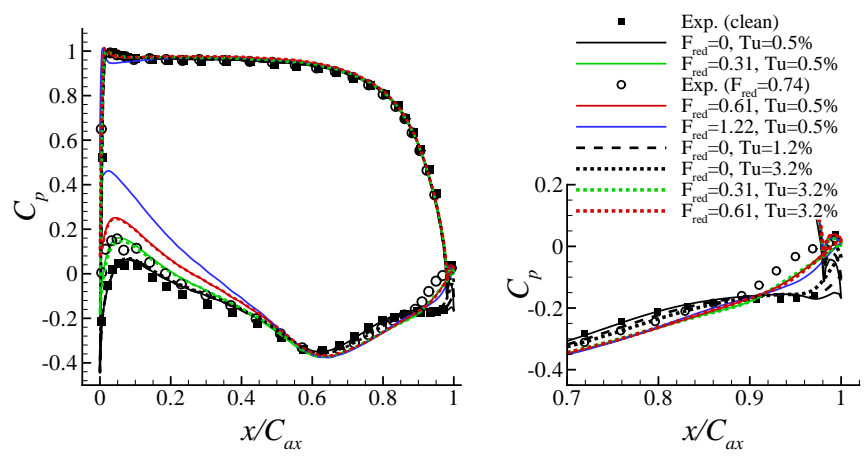

(a)
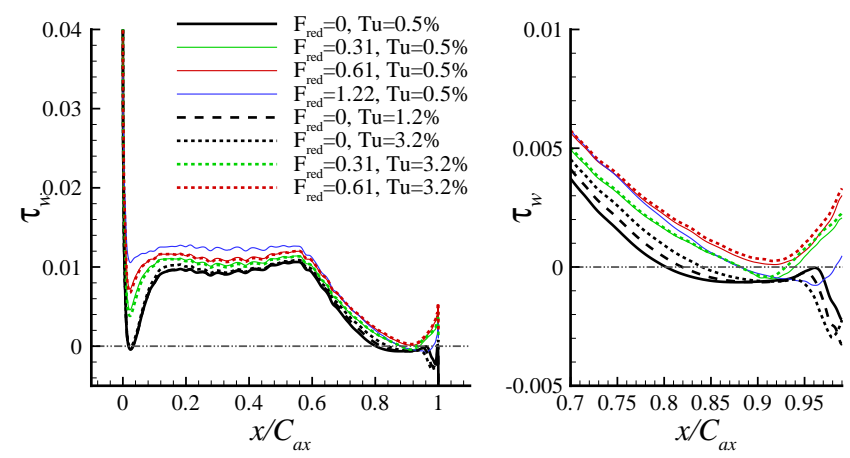

(b)

FIGURE 4: PRESSURE COEFFICIENT (a) AND WALL SHEAR DISTRIBUTION (b) ON THE BLADE SURFACE AT $R e_{2 i s}=60,000$. HERE, $C_{a x}$ IS THE AXIAL CHORD LENGTH.

with the experimental data at the clean (steady) and $F_{\text {red }}=0.74$ cases, respectively [6].

At $F_{r e d}=0$ with $T u=0.5 \%$, the plateau near the trailing edge indicates a laminar separation bubble. For $F_{\text {red }}=0$ with $T u=1.2 \%$ and $T u=3.2 \%$, the separation bubble shrinks and reattachment appears to occur further upstream with pressure rising to a larger value. This is confirmed in Fig. 4(b) showing that the separation point, where the sign of $\tau_{w}$ changes, moves from $x / C_{a x}=0.8$ in the clean case to $x / C_{a x}=0.84$ in the $T u=3.2 \%$ case. It should be noted that in Fig. 4 , there is a smallscale separation bubble near the leading edge at $x / C_{a x} \approx 0.02$. Due to the movement of the bars, the cascade inlet angles measured at $x / C=-0.3$ changes to $43.8^{\circ}$ for $F_{\text {red }}=0.31,41.7^{\circ}$ for $F_{\text {red }}=0.61$ and $36.8^{\circ}$ for $F_{\text {red }}=1.22$, which leads to the differences in $C_{p}$ and $\tau_{w}$ on the suction side as shown in Fig. 4(a) and Fig. 4(b). Aft of the suction peak, it is clear in Fig. 4(b) that in the mean sense the incoming wakes suppress the separation bubble.

Fig. 5 shows the Reynolds number effect on the pressure coefficient and the wall shear $\tau_{w}$. The main difference due to the Reynolds number increase is the pressure recovers more quickly at $R e_{2 i s}=100,000$ than at $R e_{2 i s}=60,000$. Looking at Fig. 5(b), at $R e_{2 i s}=100,000$ the leading edge separation bubble in the clean case becomes remarkably large, and at the trailing edge the flow separation is delayed. In the case at $R e_{2 i s}=100,000$ with $T u=3.2 \%$, the leading edge separation bubble shown by the negative $\tau_{w}$ shrinks, which is related to the earlier onset of the reattachment when the separated shear layer experiences larg-
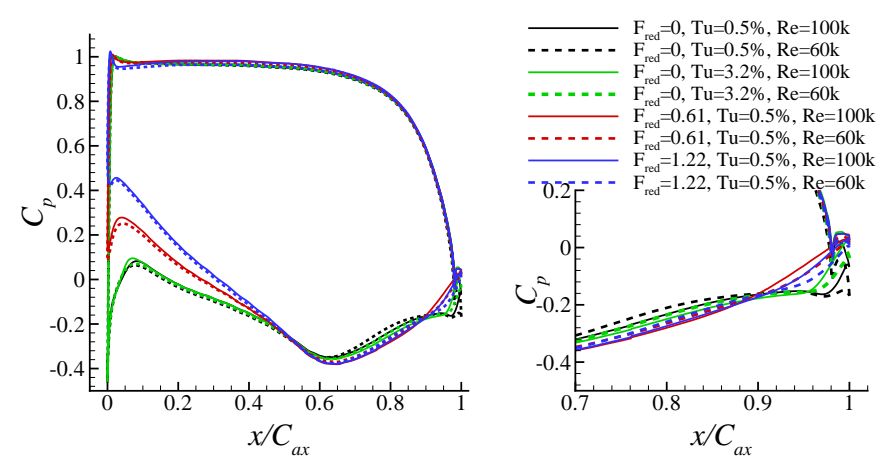

(a)
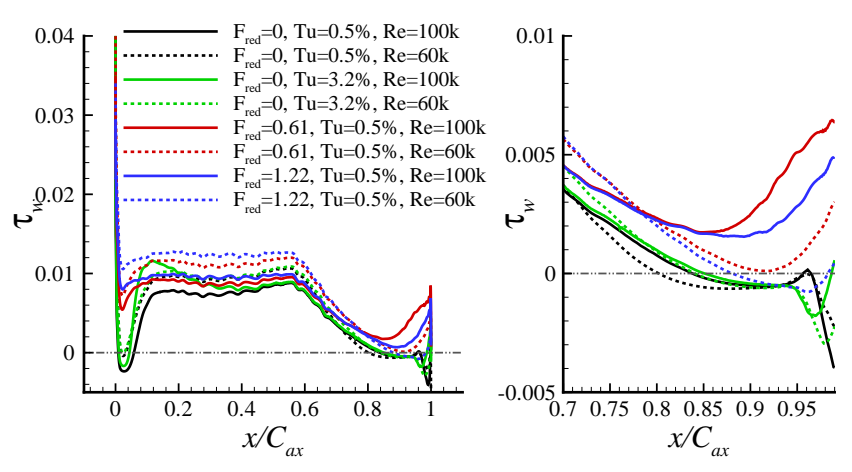

(b)

FIGURE 5: COMPARISON OF $R e_{2 i s}=60,000$ and $R e_{2 i s}=100,000:$ (a) PRESSURE COEFFICIENT AND (b) WALL SHEAR DISTRIBUTION.

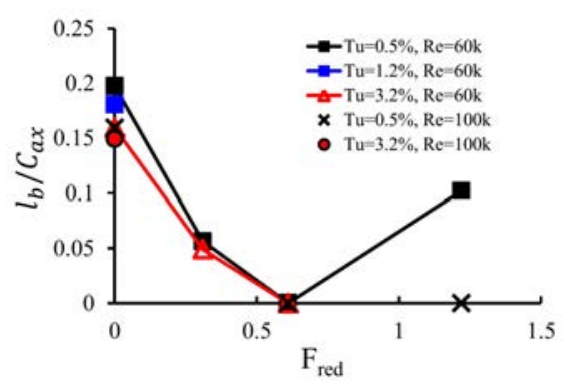

(a)

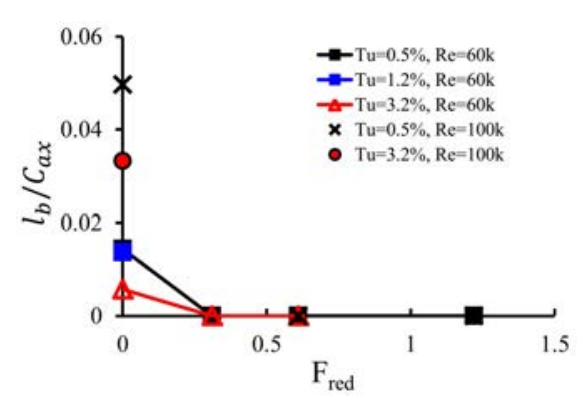

(b)

FIGURE 6: LENGTH OF SEPARATION BUBBLE: (a) TRAILING EDGE SEPARATION BUBBLE; (b) LEADING EDGE SEPARATION BUBBLE. 
er disturbances. Due to the favorable pressure gradient, these disturbances quickly decay, so the wall shear $\tau_{w}$ distribution approaches that of the clean case. For $F_{\text {red }}=0.61$ and $F_{\text {red }}=1.22$ at $R e_{2 i s}=100,000$, the wall shear profiles remain positive, indicating the boundary layers are fully attached.

The length of the separation bubbles $l_{b} / C_{a x}$ from all cases are summarized in Fig. 6. Looking at Fig. 6(a), basically the background turbulence helps to suppress the trailing edge separation bubble, especially for the cases without incoming wakes ( $F_{\text {red }}=0$ at both Reynolds numbers). The change of the trailing edge bubble size by the incoming wakes is pronounced. In the cases at $R e_{2 i s}=60,000$ without background turbulence, the bubble shrinks $75 \%$ at $F_{\text {red }}=0.31$ and is suppressed completely at $F_{\text {red }}=0.61$; however the bubble grows again at $F_{\text {red }}=1.22$. It is interesting to observe that the suction side bubble size at $F_{\text {red }}=1.22$ is similar to that at $F_{\text {red }}=0, T u=3.2 \%$. This can be explained by looking at Fig. 3: at $F_{\text {red }}=1.22$ (the highest reduced frequency) the incoming wakes merge and the boundary layer hardly perceives any discrete wake effect, rather a strong background turbulence intensity like the one at $F_{\text {red }}=0$, $T u=3.2 \%$. In the cases at $R e_{2 i s}=100,000$ with incoming wakes (i.e. $F_{\text {red }}=0.61$ and 1.22 ), the boundary layers become fully attached. As shown in Fig. 6(b), it is worth noting that remarkable leading edge separation bubble occurs in the cases at $F_{\text {red }}=0$ due to the higher inflow angles. The variation of bubble size is directly related to the profile loss generation which will be further discussed later.

\section{Phase-Locked Averaged Flow Field}

To better understand the physical processes in the boundary layer subjected to the incoming wakes, the phase-averaged statistics have been collected over 25 bar passing periods for the cases with incoming wakes. Each period is divided into 10 equal phases. The phase $\phi$ is defined by $\phi=T / T_{0}-n$, where $n$ is an integer such that $0 \leq \phi<1$, where $T_{0}$ is the bar passing period.

Space time view of the boundary layer Here, three typical cases with incoming wakes at $F_{\text {red }}=0.31,0.61$ and 1.22 ( $T u=0.5 \%$ and $\left.R e_{2 i s}=60,000\right)$ are presented to show the spacetime variation of the boundary layer. Fig. 7 shows the space-time diagrams of momentum thickness $\theta$ with $s$ denoting the surface length measured from the leading edge where $s=0$. The negative and positive values denote pressure and suction sides, respectively. The variation of momentum thickness $\theta$ and wall shear stress $\tau_{w}$ at the trailing edge $(s \approx 1.308)$ are also shown.

Looking at Fig. 7(a) and Fig. 7(b), the momentum thickness in the favorable pressure gradient region $\left(x / C_{a x}=0-0.6\right)$ on the suction side drops due to the passing of the incoming wakes. The regions of negative $\tau_{w}$ values are marked in dashed lines showing the space-time evolution of the separation bubbles. At $F_{\text {red }}=0.31$ the negative $\tau_{w}$ regions on the suction side are much larger than those of the $F_{r e d}=0.61$ case, suggesting the separation bubble at $F_{\text {red }}=0.31$ is bigger in size with a longer life time. On the pressure side, the boundary layer is mostly attached, but a small-scale separation occurs at $F_{\text {red }}=0.31$ due to the variation of instantaneous incidence angle. These two cases demonstrate that the unsteady wakes periodically reduce the extent of
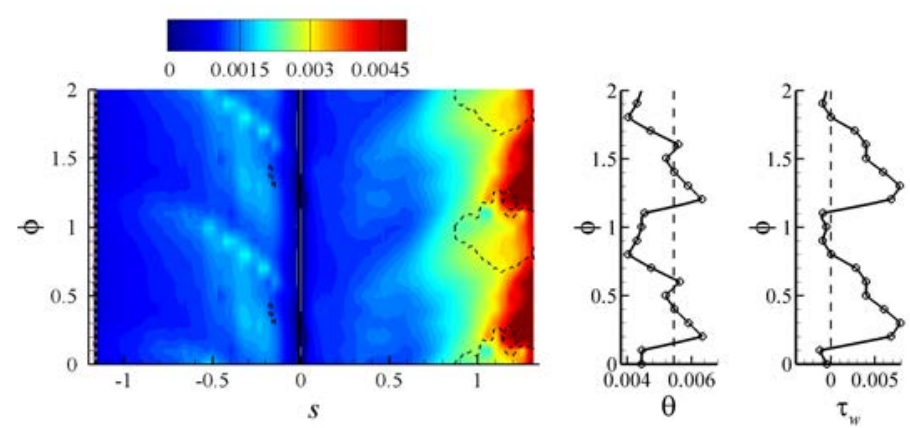

(a)
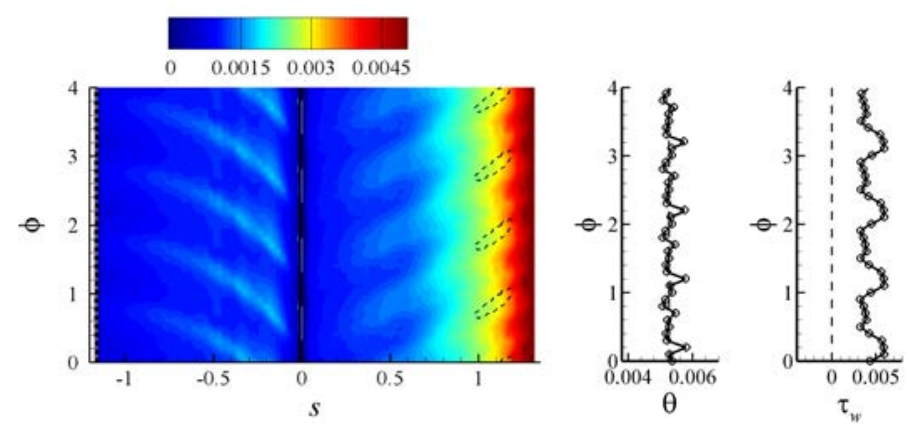

(b)
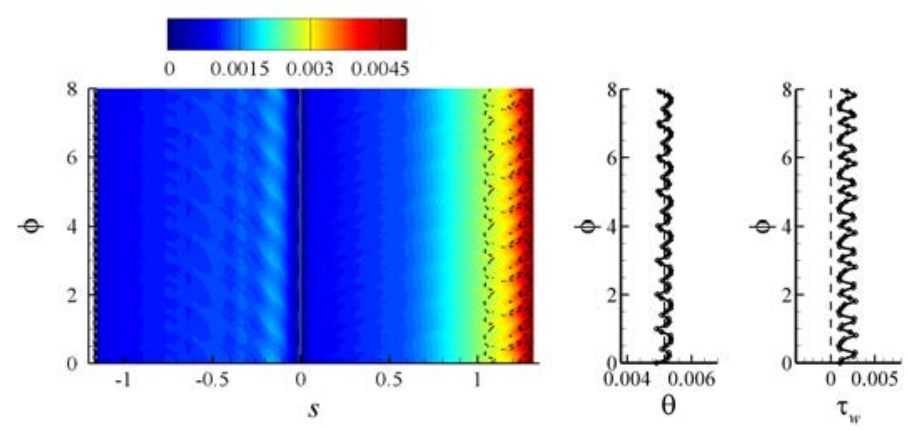

(c)

FIGURE 7: SPACE-TIME DIAGRAMS OF MOMENTUM THICKNESS $\theta$ AND THE VARIATION AT THE TRAILING EDGE $(s \approx 1.308)$ : (a) $F_{r e d}=0.31, T u=0.5 \%, R e_{2 i s}=60,000$; (b) $F_{\text {red }}=0.61, T u=0.5 \%, R e_{2 i s}=60,000$; (c) $F_{r e d}=1.22$, $T u=0.5 \%, R e_{2 i s}=60,000$.

the separation bubble, which is consistent with what we see in the flow field visualization in Fig. 2. At the trailing edge, it can be seen that at lower reduced frequency $F_{\text {red }}=0.31$, the momentum thickness and $\tau_{w}$ vary more widely than at $F_{\text {red }}=0.61$. The momentum thickness is elevated due to the reattachment.

By contrast, in Fig. 7(c), the variation of momentum thickness at $F_{\text {red }}=1.22$ subjected to the wake passing is small. The separation bubble occurs at all phases and the amplitude of $\tau_{w}$ fluctuation at the trailing edge is weakened. This is because the wakes generated by four moving cylinders have considerably mixed and decayed, as aforementioned. As a result, there is no significant change in the separation bubble size and the boundary layer status at the trailing edge throughout the bar-passing cycle. 


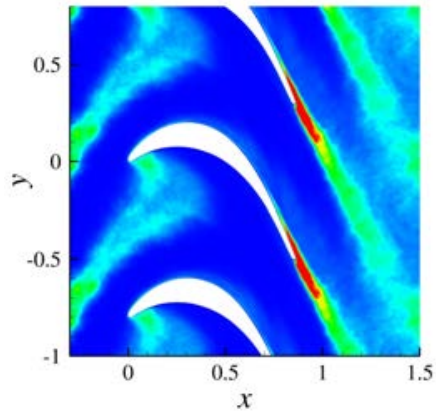

(a)

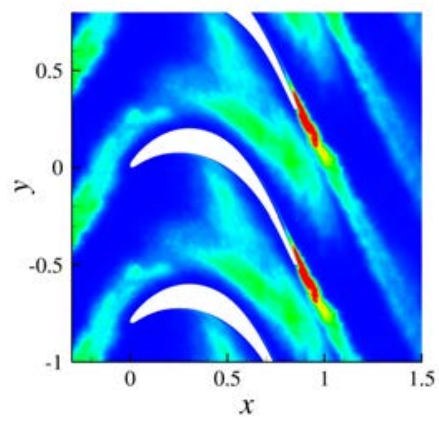

(c)

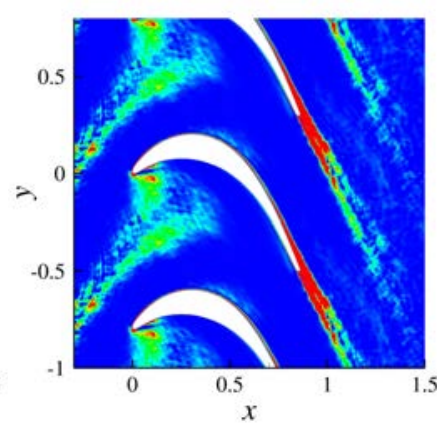

(b)

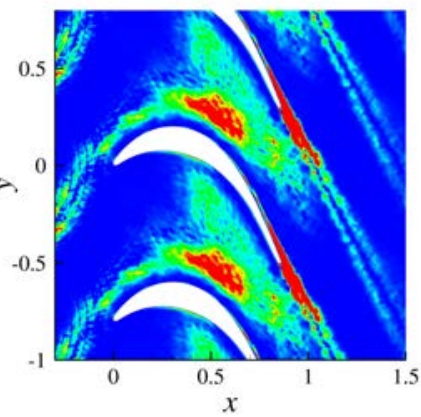

(d)
FIGURE 8: THREE PHASE-AVERAGED SNAPSHOTS OF TURBULENT KINETIC ENERGY (TKE) (a,c) AND TKE PRODUCTION TERMS $(b, d)$ WITH THE CONTOUR LEVELS BETWEEN 0 (BLUE) AND 0.05 (RED): 0.4 AND 0.8 $\left(F_{r e d}=0.31, T u=0.5 \%\right.$ and $\left.R e_{2 i s}=60,000\right)$.

Turbulent flow field For $F_{\text {red }}=0.31 \quad(T u=0.5 \%$, $\left.R e_{2 i s}=60,000\right)$, Fig. 8 shows two snapshots of the phaseaveraged turbulent kinetic energy (TKE) and TKE production term: $\phi=0.4$ and $\phi=0.8$. At $\phi=0.4$, the incoming wakes decay and start to distort when they go into the blade passage and the peak TKE value in the wake is $\sim 0.01 U_{\infty}^{2}$. Due to the unsteady flow downstream of the trailing edge, the TKE in the blade wake near the trailing edge reaches $0.1 U_{\infty}^{2}$. As shown in Fig. 8(b), the maximum TKE production in the incoming wake is around 0.01 , and the value in the blade wake is about $O(1.0)$, corresponding to the high turbulent kinetic energy distribution in these regions. Fig. 8(c) shows at $\phi=0.8$, the incoming wake convects further downstream and highly distorts in the variablearea blade passage, resulting in the high TKE region in the blade passage, where the peak value reaches $0.02 U_{\infty}^{2}$. Correspondingly, the TKE production significantly strengthens because of mean velocity gradient, and reaches as high as 0.05 in the blade passage as shown in Fig. 8(d).

Fig. 9 shows two snapshots of the phase-averaged TKE and TKE production term for the case at $F_{\text {red }}=0.61$ with $T u=0.5 \%$ at $R e_{2 i s}=60,000$. In Fig. $9(\mathrm{a}, \mathrm{c})$, three of the incoming wakes are labelled. From $\phi=0-0.4$, it displays the process of the wake distortion and convection in the variable-area blade passage. Consistent with the high TKE region, the region where TKE production term is enhanced can be found in the passage as

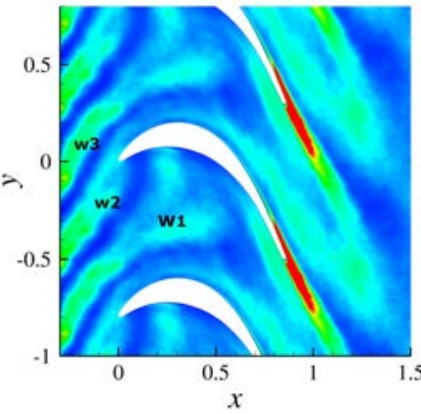

(a)

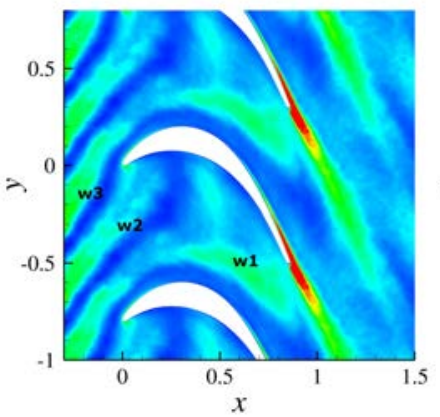

(c)

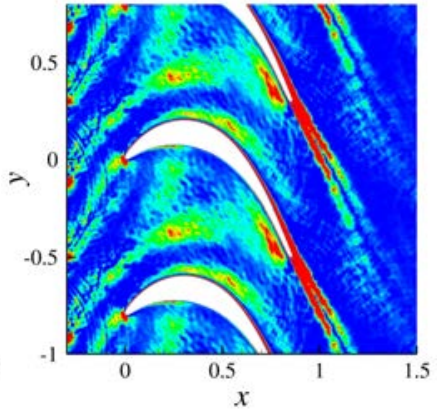

(b)

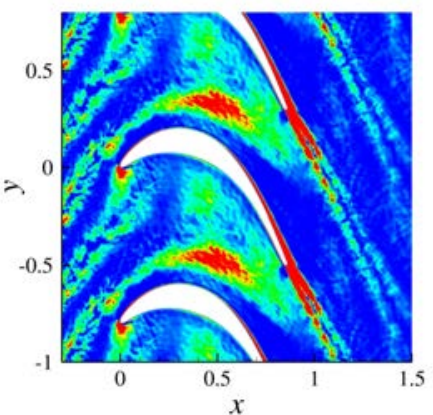

(d)
FIGURE 9: THREE PHASE-AVERAGED SNAPSHOTS OF TURBULENT KINETIC ENERGY (TKE) $(\mathrm{a}, \mathrm{c})$ AND TKE PRODUCTION TERMS (b,d) WITH THE CONTOUR LEVELS BETWEEN 0 (BLUE) AND 0.05 (RED): $\phi=0$ and 0.4 $\left(F_{\text {red }}=0.61, T u=0.5 \%\right.$ AND $\left.R e_{2 i s}=60,000\right)$.

shown in Fig. 9(d).

In general, the turbulence production rate in the incoming wake can reach levels similar to those in the trailing edge wakes, but only at selected times. This has to be kept in mind in the next section when analyzing the unsteady loss generation mechanism driven by the incoming wakes.

\section{Summary of Losses}

Conventional loss calculation Fig. 10(a) shows the kinetic loss profile $\Omega$, defined as $\left(p_{t, 1}^{A}-p_{t, 2}\left(y^{*}\right)\right) /\left(p_{t, 1}^{A}-p_{2}^{A}\right)$, at $R e_{2 i s}=60,00040 \% C$ downstream the trailing edge along the non-dimensional pitchwise direction $y^{*}=\left(y-y_{L E, 0}\right) /\left(y_{L E, 1}-\right.$ $\left.y_{L E, 0}\right)$. The profile shape and absolute value are a challenge for traditional CFD [16]. The $F_{\text {red }}=0 T u=0.5 \%$ case compares favorably with experiments, the wake appears wide due to the open separation evidenced in Fig. 4. At an inlet turbulence intensity of $1.2 \%$ the bubble tends to shorten, but it is only at $T u=3.2 \%$ that the separation closes at the trailing edge and the stagnation pressure defect reduces on the SS side portion of the profile. The low $F_{\text {red }}=0.3$ case is investigated without and with background turbulence intensity. In both cases, $T u=1.2 \%$ and $3.2 \%$, Fig. 4 suggests the incoming wakes are enough to reduce the time-averaged boundary layer separation (see Fig. 6). Despite the differences in the $C_{p}$ profile driven by incoming wakes, it is notable that the $F_{\text {red }}=0, T u=3.2 \%$ shows a kinetic loss 


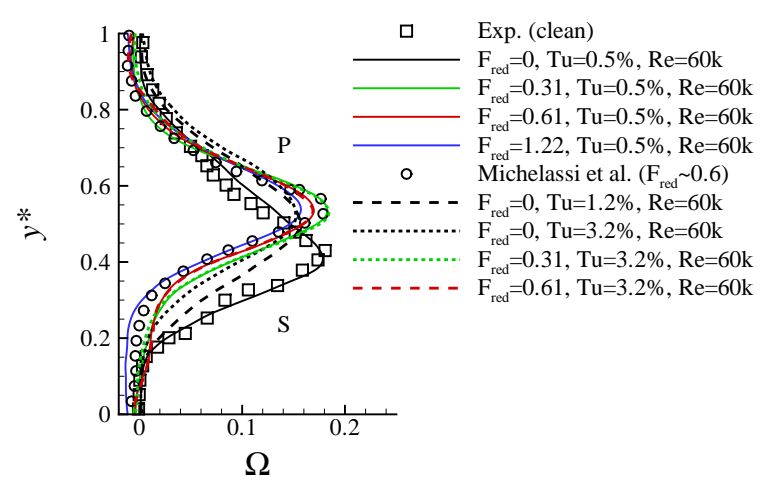

(a)

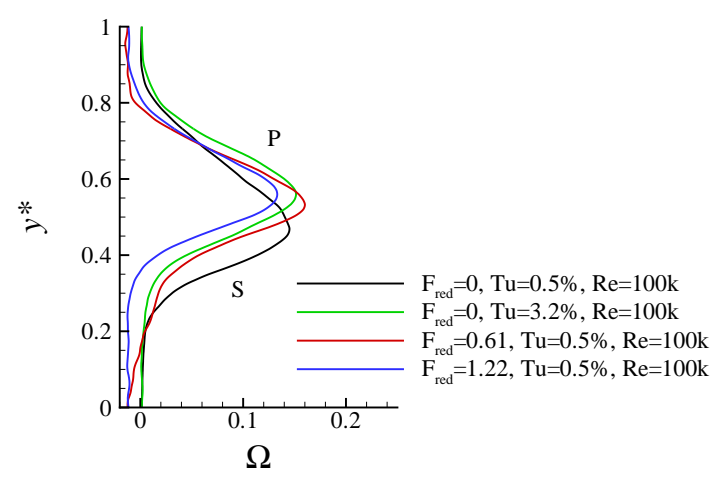

(b)

FIGURE 10: COMPARISON OF THE WAKE STAGNATION PRESSURE LOSS PROFILES WITH REFERENCE DATA: (a) $R e_{2 i s}=60,000$ AND (b) $R e_{2 i s}=100,000$. HERE, 'P' DENOTES PRESSURE SIDE AND 'S' SUCTION SIDE.

profile quite similar to the $F_{\text {red }}=0.3$ case. Increasing the reduced frequency to 0.6 , without and with inlet turbulence does not alter the $\Omega$ profile shape, although the wake becomes slightly shallower and narrower. The computed profile agrees well with the incompressible one by Michelassi et al. [13]. Increasing $F_{\text {red }}$ further to 1.22 does not produce additional changes. At $R e=100,000$ (Fig. 10(b)) the $F_{\text {red }}=0$ shows the same effect of $T u$ seen at the lower Re. Again, a large turbulence intensity without incoming wakes can give a loss profile shape similar to that at $F_{\text {red }}=0.61$ and 1.22 .

Fig. 11 shows the mixed-out loss, defined as $\omega^{M}=\left(p_{t, 1}^{M}-\right.$ $\left.p_{t, 2}^{M}\right) /\left(p_{t, 1}^{M}-p_{2}^{M}\right)$ versus $F_{\text {red }}$. Here $M$ denotes a mixed-out quantity. The $T 106$ case is stalled at $F_{\text {red }}=0$. The suction side separation reduction justifies the strong loss drop with increasing $F_{\text {red }}$ or $T u$ (see for example how the $F_{\text {red }}=0.3$ corresponds to the $F_{\text {red }}=0, T u=3.2 \%$ case). The inlet turbulence intensity is discovered to reduce losses only without incoming wakes, by reducing the separation bubble, while it does increase losses when combined with $F_{\text {red }}>0$, possibly due to the interaction of wakes and homogeneous turbulence. The same trend holds for the two $R e_{2 i s}$ levels.

Loss Breakdown In presence of large scale deterministic unsteadiness provoked by incoming wakes losses generate

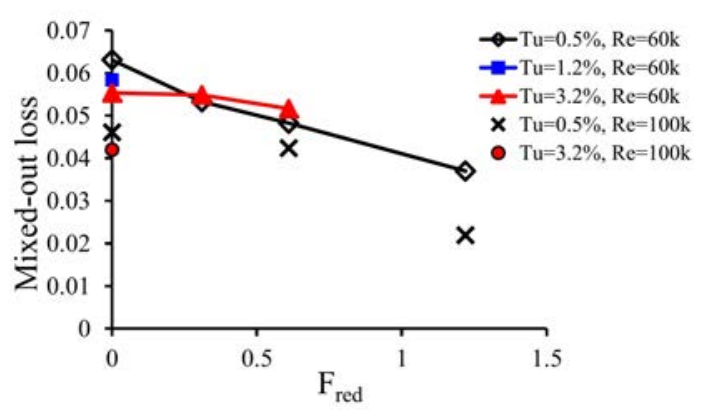

FIGURE 11: STAGNATION PRESSURE LOSSES CALCULATED WITH MIXED-OUT QUANTITIES.

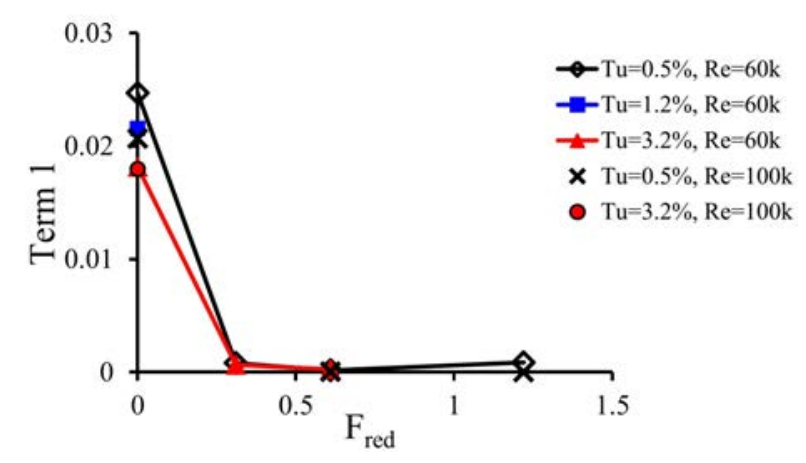

(a) THE BASE PRESSURE LOSS.

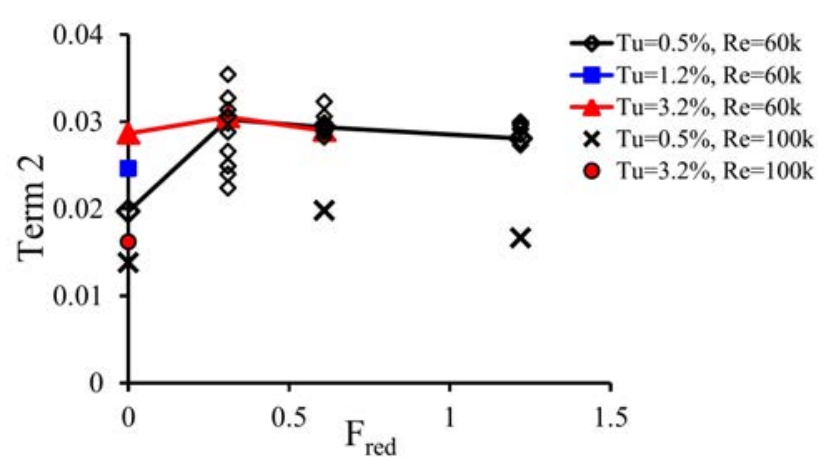

(b) THE MIXED-OUT LOSS OF THE BOUNDARY LAYER (OR THE MOMENTUM DEFICIT LOSS).

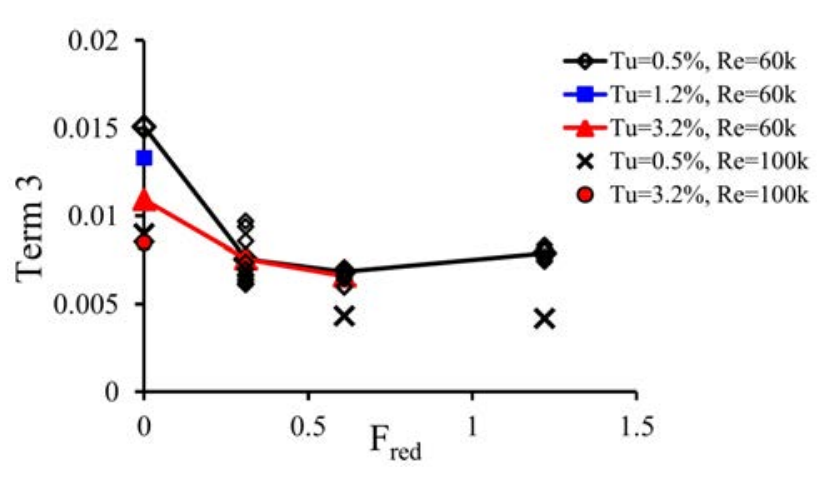

(c) THE COMBINED BLOCKAGE LOSS.

FIGURE 12: TERMS OF THE TRAILING EDGE PROFILE LOSS IN EQ. (1) SUGGESTED BY DENTON [19]. 


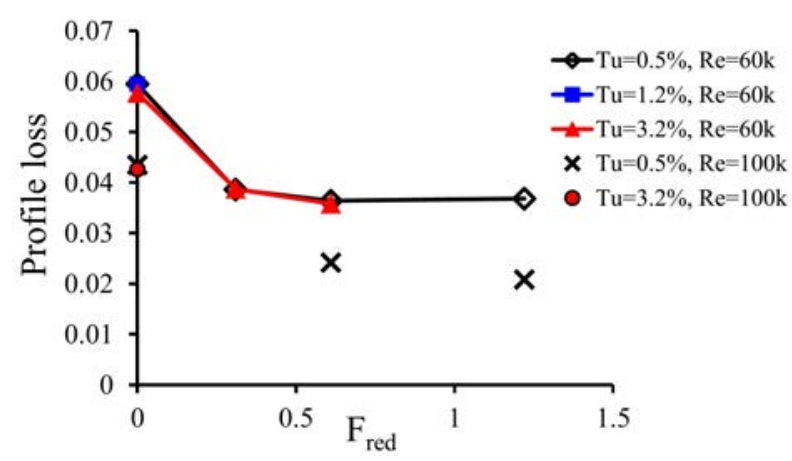

(a) DENTON'S PROFILE LOSS.

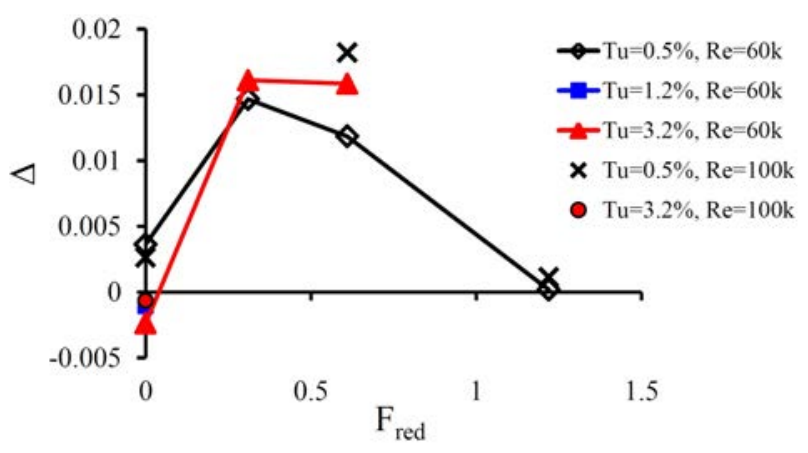

(b) DEVIATION OF MIXED-OUT LOSS AND DENTON'S PROFILE LOSS.

FIGURE 13: DENTON'S TRAILING EDGE LOSS COEFFICIENT (a) AND THE DIFFERENCE FROM MIXED-OUT LOSSES (b).

inside and outside the boundary layer. This chapter attempts to quantify these losses with the help of the DNS database.

In the classical lecture by Denton [19], the loss due to the mixing out of a wake behind a trailing edge is derived as

$$
\zeta=-\frac{C_{p b} t}{s_{b} \cos \left(\beta_{2}\right)}+\frac{2 \theta}{s_{b} \cos \left(\beta_{2}\right)}+\left(\frac{\delta^{*}+t}{s_{b} \cos \left(\beta_{2}\right)}\right)^{2}
$$

where $t$ is the thickness of the trailing edge, and $\delta^{*}$ and $\theta$ are the displacement and momentum thickness, respectively. The base pressure coefficient $C_{p b}$ is defined by

$$
C_{p b}=\frac{P_{b}-P_{s}}{p_{t, 1}^{M}-p_{2}^{M}}
$$

where $P_{b}$ is the base pressure. In the original theory, $P_{s}$ is the average pressure acting on the suction surface downstream of the throat, and it assumes that this is the same as the pressure far downstream, $P_{2}$ [19]. In the present work, we use the throat pressure as $P_{S}$ [20]. Eq. (1) is computed term by term by using the time averaged flow fields of the 12 different DNS results.

In Eq. (1), the first term is the loss due to the low base pressure at the trailing edge. In presence of large trailing edge separations, Denton suggested two corrections [19]: first, to use $\left(t+\delta^{*}\right)$ instead of $t$ in the base pressure term of Eq. (1); second, to apply the low base pressure obtained from the whole separated region. Fig. 12(a) summarizes the results. Since the base pressure loss is associated with the separated flow, term 1 exhibits a similar trend as that of the trailing edge separation bubble length (see Fig. 6(a)). In the cases at $F_{\text {red }}=0$ with larger separations $(T u=0.5 \%-3.2 \%)$, the base pressure losses are notably high, while for the cases with attached boundary layers, e.g. $F_{\text {red }}=0.61$ with $T u=0.5 \%$ at $R e_{2 i s}=60,000$, the base pressure losses approach zero.

The second term in Eq. (1) is the boundary layer momentum deficit loss, derived from the momentum thickness on the suction side just before the trailing edge [4]. Fig. 12(b) shows low values of term 2 at $F_{\text {red }}=0$ as, with large separations the momentum deficit is small. At $R e_{2 i s}=60,000$ with wakes, regardless of the incoming turbulence level, term 2 exhibits a nearly constan$\mathrm{t}$ value of 0.03 , as the boundary layers are mostly attached. At $R e_{2 i s}=100,000$, this value drops to $\sim 0.02$. In order to quantify term 2 unsteadiness, 10 phase-averaged points for $F_{\text {red }}=0.31$ to 1.22 with $T u=0$ at $R e_{2 i s}=60,000$ are added into Fig. 12(b) as diamonds around their time-mean. At $F_{\text {red }}=0.31$, the momentum deficit losses vary widely, but, as the reduced frequency increases, this variation becomes less pronounced, suggesting a wake-to-wake interaction that reduces the discrete nature of the incoming disturbances.

The third term in Eq. (1) accounts for blockage, and it becomes important only in presence of stall. Fig. 12(c) shows high blockage losses in presence of separations, presenting the same trend as the evolution of the separation bubble and term 1 . Note also that the scatter of the phase-averaged data points (diamonds) of term 3 is consistent with that of term 2 .

The summation of the three terms is given in Fig. 13(a). For the attached case and in presence of wakes, the momentum deficit term is the dominant source of profile losses. The case at $F_{\text {red }}=0$ and $R e_{2 i s}=60,000$ is the most sensitive to the background turbulence, and for $F_{\text {red }}>0$ the incoming wakes significantly reduce the profile losses. Increasing $F_{\text {red }}$ above 0.61 does not reduce losses any further.

To identify the impact of incoming wakes, the profile losses of Fig. 13(a) are subtracted from the mixed-out losses of Fig. 11. The result, $\Delta$, is shown in Fig. 13(b). Observe the comparison assumes the inlet mixed-out stagnation pressure as reference.

The difference is small (less than $0.5 \%$ ) for $F_{\text {red }}=0$, implying that the formulation by Denton (1) performs well without incoming wakes. It was found possible to bring this difference almost to zero by adding $\left(\delta_{L E}^{*}+t\right)^{2} / w^{2}$ to term 3 to include the effect of the leading edge separation (see Fig. 6 (b)), where $\delta_{L E}^{*}$ is the displacement thickness near the leading edge separation bubble). Deviations peak at $F_{\text {red }}=0.31$ and drop almost to zero at $F_{\text {red }}=1.22$, while the inlet turbulence intensity does seems to have a weak effect. The $\Delta$ is likely to be caused by three effects: (a) mixing-out of incoming wakes at constant area upstream of leading edge, $(b)$ mixing-out of incoming wakes in the blade passage, i.e. at variable area, and $(c)$ unsteady boundary layer losses.

To quantify the impact of $(a)$ we defined $\omega^{A}=\left(p_{t, 1}^{A}-\right.$ $\left.p_{t, 2}^{M}\right) /\left(p_{t, 1}^{A}-p_{2}^{M}\right)$, where $A$ denotes a mass-flow averaged quantity, and plot $\eta_{1}=\omega^{A}-\omega^{M}$. The small values of $\eta_{1}$ suggest 
the constant-area mixing of the wake upstream of the leading edge has limited effect. The effect of $(b)$, the variable area wakemixing, is computed by defining $\eta_{2}=\left(p_{t, L E}^{A}-p_{t, T E}^{A}\right) /\left(p_{t, L E}^{A}-\right.$ $\left.p_{T E}^{A}\right)$, in which the static and stagnation pressures are averaged along two sections corresponding to the leading $(L E)$ and trailing ( $T E$ ) edges, as shown in the sketch of Fig. 14. The boundary layers were cut off to include only the flow core. We tested different methods to remove boundary layer effects from $\eta_{2}$, and the trend remained the same. The comparison of $(a)$ and $(b)$ suggests that the losses associated to the costant area mixing of incoming wakes before the leading edge is much smaller than the losses caused by the mixing out of the same wakes at variable area from leading to trailing edge.

Fig. 3 and Fig. 14 also suggest the wake distortion losses $\eta_{2}$ are high as long as the wakes stay discrete and the waketo-wake interaction is weak at $F_{\text {red }}=0.31-0.61$. Notably, $\eta_{2}$ has almost the same value in the absence of wakes and when wakes merge at $F_{\text {red }}=1.22$, thereby suggesting that when wakes interact and merge they tend to provoke losses similar to those of a background turbulence.

The remaining difference $(c)$ between $\Delta$ and $\eta_{2}$ can be attributed to unsteady boundary layer effects triggered by the discrete incoming wakes. This difference is small at $F_{\text {red }}=0$, without wakes, and 1.22 when wakes merge. The deviation peaks at $F_{\text {red }}=0.31$, when the largest intermittent boundary layer separation occurs, and reduces at $F_{\text {red }}=0.61$ when the intermittent separation is minimum (see Fig. 6).

\section{Validity of the Boussinesq viscosity assumption}

Both DNS and LES remain prohibitively expensive to be considered for design of turbomachinery. RANS calculations therefore still play a major role in this field but do rely on the modelling of the velocity fluctuation correlations. In many popular RANS closures these fluctuation correlations are modelled using the Boussinesq approximation (eddy viscosity assumption). In the case of compressible flows the Boussinesq approximation reads

$$
\tau_{i j} \equiv \tau_{b, i j}=\underbrace{2 \mu_{t}\left[\widetilde{S}_{i j}-\frac{1}{3} \widetilde{S}_{i i} \delta_{i j}\right]}_{\text {anisotropic }}-\underbrace{\frac{2}{3} \bar{\rho} k \delta_{i j}}_{\text {isotropic }}
$$

where the isotropic part is given by the turbulent kinetic energy $k=-\tau_{i i}$ and the anisotropic part is modelled using the strain rate. Both $k$ and $\mu_{t}$ are solutions of particular turbulence models, thus no general statements on their validity for all Boussinesq-based turbulence models can be made. In contrast, using the traceless stress $\tau_{b, i j}^{*}=\tau_{b, i j}+2 / 3 \bar{\rho} k \delta_{i j}$ and strain $\widetilde{S}_{i j}^{*}=\widetilde{S}_{i j}-1 / 3 \widetilde{S}_{i i} \delta_{i j}$ tensors, Eqn. (3) can be rewritten as $\tau_{b, i j}^{*}=2 \mu_{t} \widetilde{S}_{i j}^{*}$, showing that the anisotropic part of the modelled stress and the anisotropic part of the traceless mean strain rate tensor have a linear relation due to the multiplication by a 'scalar' turbulence viscosity $\mu_{t}$. This linear coupling applies to all Boussinesq based turbulence models and therefore general statements can be made for this part. It has been shown that this linear coupling of Reynolds stress and

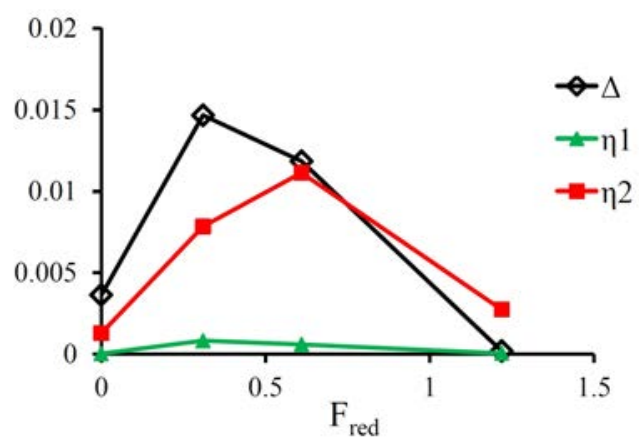

(a)

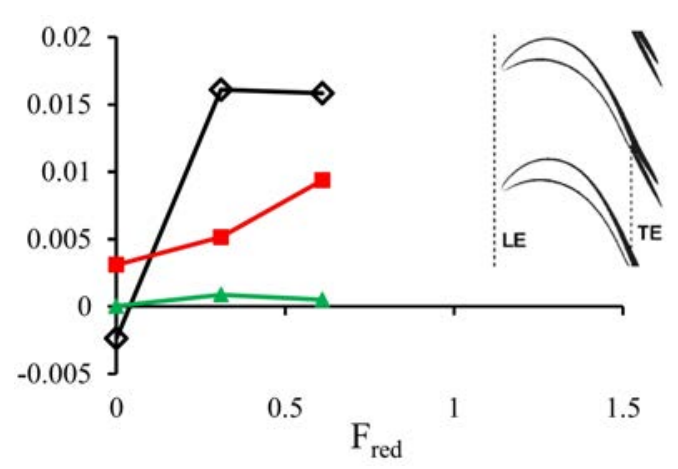

(b)

FIGURE 14: COMPARISON OF THE DEVIATION $\Delta$, WAKE MIXING $\eta_{1}$ AND DISTORTION LOSSES $\eta_{2}$ : (a) $T u=0.5 \%$, $R e_{2 i s}=60,000$; (b) $T u=3.2 \%, R e_{2 i s}=60,000$. THE SKETCH SHOWS THE LOCATIONS OF THE PROFILES EXTRACTED OUTSIDE OF THE BOUNDARY LAYER.

mean strain is a poor model for a range of flows, partly due to larger time and length scales in turbulence compared to molecular motion, which served as basis of the Boussinesq approximation [21]. To what extent the Boussinesq approximation is valid in LPT flows is not entirely clear and will be investigated in the following. The modeling error made by assuming a linear stressstrain relationship can be quantified in terms of the Frobenius norm of the difference of the stress tensor obtained from DNS and the Boussinesq modeled stress tensor

$$
e_{t}=\left\|\tau_{i j}^{*}-\tau_{b, i j}^{*}\right\|=\left\|\tau_{i j}^{*}-2 \mu_{t} \widetilde{S}_{i j}^{*}\right\|
$$

From DNS $\tau_{i j}^{*}$ and $\widetilde{S}_{i j}^{*}$ are available but the turbulence viscosity is not a flow quantity and has to be obtained otherwise. Two different definitions are chosen and compared in this work. In order to get the minimum possible error a turbulence viscosity is defined setting the derivative of $e_{t}$ with respect to $\mu_{t}$ to zero, which after rearranging yields

$$
\mu_{t, o p t}=0.5\left(S_{k l}^{*} \tau_{k l}^{*}\right) / S_{k l}^{* 2}
$$

The importance of $\mu_{t, o p t}$ is that with respect to the above error definition no turbulence model based on the Boussinesq approximation can perform better and the error stems only from 


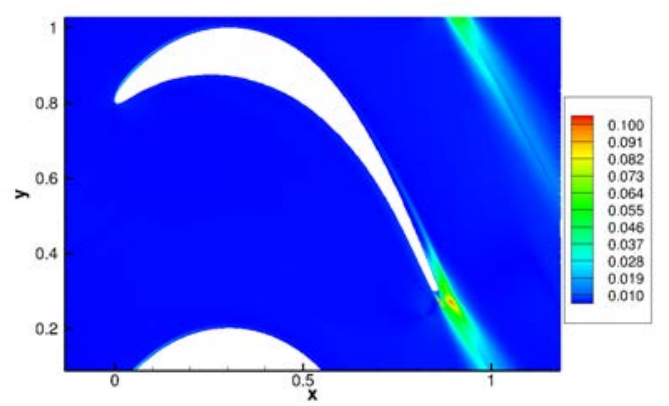

FIGURE 15: MINIMUM REYNOLDS STRESS ERROR $e_{t, \text { min }}$ USING BOUSSINESQ APPROXIMATION AT $R e_{2 i s}=60,000$ AND $T u=3.2 \%$.

the requirement of linear stress-strain coupling. But this turbulence viscosity is a mathematical minimum rather than based on physical modelling and thus cannot be applied directly to RANS calculations. Therefore, $\mu_{t, k \varepsilon}=c_{\mu} k^{2} \varepsilon^{-1}$ with $c_{\mu}=0.09$ as used by $k-\varepsilon$ models can be used as a second choice for $\mu_{t}$, as both $k$ and $\varepsilon$ are available from DNS data.

In Fig. 15 the minimum obtainable error using the Boussinesq approximation with $\mu_{t, o p t}$ is plotted for a case at an isentropic exit Reynolds number of 60,000 and approximately 3.2\% inlet turbulence. At the inlet the turbulence is nearly isotropic and therefore, as this error only considers the anisotropic part of the Reynolds stress, the error is close to zero. In the blade channel non-isotropic strain is present, leading to production of non isotropic Reynolds stresses. However, the absolute error remains small in this region. The largest error regions can be identified as being close to the leading and trailing edges of the blade. In case of the leading edge, the increased error is due to the incoming turbulence interacting with the strain close to the boundary layer, resulting in production and distortion of turbulence that is not appropriately modelled. The large absolute errors observed in the vicinity of the trailing edge might affect the breakdown of the vortices shed by the trailing edge and will compromise an accurate representation of the wake shape and dissipation.

Fig. 16 shows the modeling error using the $k-\varepsilon$ based turbulence viscosity for one of the phase averaged flow fields. In this case the error found in the bar-wake in the blade passage is about an order of magnitude smaller than in the blade-wake. The error downstream of the wake-producing bars, not seen in the figure, is of similar order to the blade-wake and highlights the expected problem of Boussinesq type turbulence models with vortex shedding. This is due to the fact that bluff body separated flows (and their vortex shedding) are significantly different to the (thin) shear layers being considered in the derivation of the Boussinesq approximation. Even though a URANS is expected to be beneficial in cases of vortex shedding there are two major uncertainties. Firstly, no clear spectral gap exists, as seen in Fig. 12 in part 1 of this paper, peaks in the spectrum due to vortex shedding and separation bubble unsteadiness are well within the turbulence frequency range. Secondly, the wake vortex breakdown and wake shape depend on the background turbulence level, as pointed out in part 1 of this paper. These two uncertainties require further investigations.

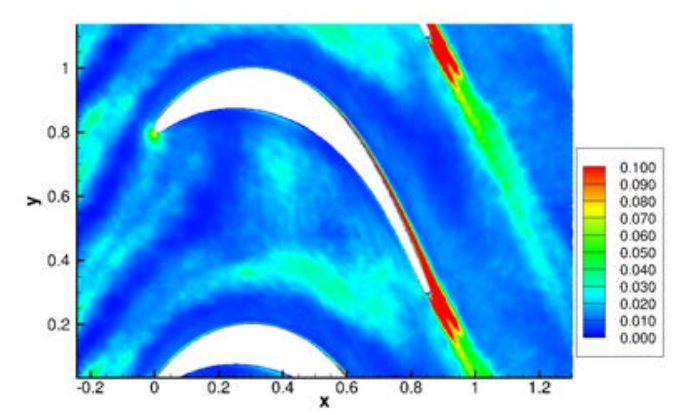

FIGURE 16: REYNOLDS STRESS ERROR $e_{t, k \varepsilon}$ USING $k-\varepsilon$ MODEL WITH THE BOUSSINESQ APPROXIMATION AT $R e_{2 i s}=60,000$ AT A REDUCED FREQUENCY OF 0.62 AT THE SAME TIME INSTANT AS IN FIG. 9(d).

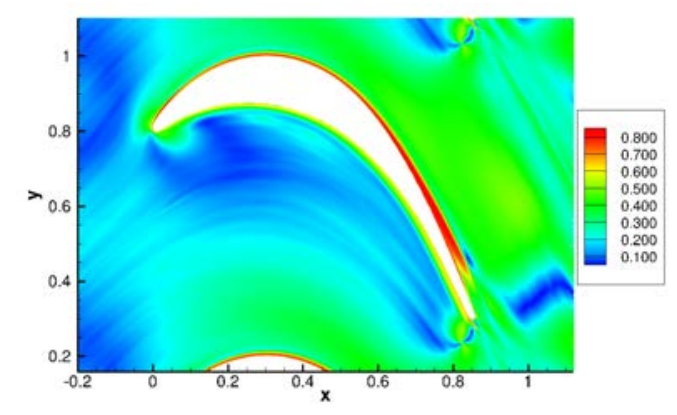

FIGURE 17: NORMALIZED REYNOLDS STRESSES ERROR USING THE BOUSSINESQ APPROXIMATION AT $R e_{2 i s}=$ 60,000 AND $T u=3.2 \%$.

By comparing different cases of the conducted Reynolds number and reduced frequency sweeps, it was found that the absolute error at the trailing edge decreases with increasing turbulence levels and increasing Reynolds number. This is most likely due to a faster breakdown of the vortex shedding into three dimensional turbulence. Further, for a reduced suction separation bubble length, the trailing edge vortex shedding is less intense. At the leading edge the error scales with turbulent intensity.

The investigation of absolute errors does not directly elucidate in which regions the Boussinesq approximation is more or less valid. A smaller absolute error might simply stem from the fact that the anisotropic part of the Reynolds stress is small and nothing has to be modelled. The validity of the Boussinesq approximation can be better shown by plotting the error normalized with the norm of the Reynolds stress, see Fig.17. This definition highlights areas where linear stress-strain coupling is not valid regardless of the magnitude of the error introduced so that areas with almost no anisotropic stress can dominate. In fact, while for the majority of the flow field the error is of the order of $20 \%$ to $40 \%$, in the boundary layers it reaches $80 \%$ even though the flow is mostly laminar. In contrast to the absolute error the normalized error at the trailing edge is close to the values of the surrounding flow field. The low error region at about $x=1$ and $y=0.3$ is due to the zero crossing in the main error component. Hence, the linear stress-strain relationship assumption is violated for large sections of the flow field, and in regions with large anisotropic 
stress contributions, i.e. at the trailing and leading edges, a large absolute error occurs.

\section{CONCLUSION}

Direct numerical simulations of the compressible flow in the T106 linear cascade have been conducted. Various environmental variables, i.e. background turbulence level, frequency of incoming wakes and Reynolds number, and a combination of these were considered for a total of 12 fully resolved simulations. The results compare favorably with the reference data in terms of the pressure distributions and wake losses.

Background turbulence and incoming wakes help to reduce the size or suppress the separation bubble. The simulations revealed that at $R e_{2 i s}=60,000$, the time averaged separation disappears at $F_{\text {red }}=0.61$, while it is present at $F_{\text {red }}=0 ., 0.31,1.22$. The evolution of the separation bubble and the boundary layer characteristics subjected to the incoming wakes are discussed by means of space-time diagrams for the cases at $T u=0.5 \%$, $R e_{2 i s}=60,000$. At low reduced frequency, $F_{\text {red }}=0.31$, the momentum thickness at the trailing edge varies more widely than at $F_{\text {red }}=0.61$ and $F_{\text {red }}=1.22$ due to the severe change of the separation bubble. It is concluded that for $F_{\text {red }}=1.22$ wakes merge and tend to behave similar to background turbulence with reduced deterministic effect.

The comparions of mixed-out losses with profile losses as defined by Denton allows discerning the losses generated in the boundary layer from those generated in the flow core by the wake mixing. The momentum deficit loss is found to be the dominant source of losses with attached boundary layer. For $T u=0.5 \%$, with the increment of reduced frequency, the mixed-out loss is reduced. Background turbulence reduces the mixed-out losses only at $F_{\text {red }}=0$, but causes extra losses at $F_{\text {red }}=0.31$ and 0.61 probably due to the wake mixing across the cascade. Denton's profile loss equation performs well without incoming wakes and with the highest $F_{\text {red }}$ of 1.22 , but shows deviations from the mixedout losses with $F_{\text {red }}=0.31-0.61$. These differences are related to the losses caused by the variable area mixing of the discrete incoming wakes into the passage, while at $F_{\text {red }}=1.22$ most of the wake mixing takes place at constant area before the leading edge. The wake distortion, as long as wakes remain discrete, plays a dominant role in the extra loss generation.

Finally, the modelling error made using the linear stressstrain coupling of the Boussinesq approximation has been investigated. This indicated where the models fail (normalized error) and to what extent (absolute error). The absolute error is highest in the trailing edge region, while the minimum achievable error is about a factor of three to four smaller than the error computing $\mu_{t}$ as in a standard $k-\varepsilon$ model with both $k$ and $\varepsilon$ from DNS data. Using a relative error definition it was also demonstrated that the linear stress-strain coupling is violated for most of the flow field but this error is only crucial in regions where a significant anisotropic stress is present.

Future analyses will focus on the incoming wake path impact on losses, and how flow core and boundary layer losses modeling can be improved in the framework of RANS.

\section{ACKNOWLEDGMENT}

The authors would like to thank General Electric Global Research for permission to publish this work. The authors are very grateful to Dr. Roderick Johnstone, Dr. Sara Delport and Dr. Rathakrishnan Bhaskaran for the valuable discussions.

\section{REFERENCES}

[1] Prakash, C., Cherry, D. G., Shin, H. W., Machnaim, J., Dailey, L., Beacock, R., Halstead, D., Wadia, A. R., Guillot, S., and Ng, W. F., 2008. "Effect of Loading Level and Distribution on LPT Losses”. ASME paper GT2008-50052.

[2] Arndt, N., 1993. "Blade Row Interaction in a Multistage Low-Pressure Turbine”. ASME Journal of Turbomachinery, 115, pp. 137-146.

[3] Halstead, D., Wisler, D., Okiishi, T., Walker, G., Hodson, H., and Shin, H., 1997. "Boundary Layer Development in Axial Compressors and Turbines: Part 1 of $4 \mathrm{C}$ Composite Picture". ASME Journal of Turbomachinery, 119, pp. 114127.

[4] Coull, J. D., Thomas, R. L., and Hodson, H. P., 2010. "Velocity Distributions for Low Pressure Turbines". ASME Journal of Turbomachinery, 132(4), p. 041006.

[5] Stadtmüller, P., and Fottner, L. "A Test Case for the Numerical Investigation of Wake Passing Effects on a HighlyLoaded LP Turbine Cascade Blade". ASME Paper GT20010311 .

[6] Stadtmüller, P., 2001. "Investigation of Wake-Induced Transition on the LP Turbine Cascade T106A-EIZ”. In DFG-Verbundprojekt Fo 136/11 Version 1.1.

[7] Michelassi, V., Wissink, J., and Rodi, W., 2003. "Direct Numerical Simulation, Large Eddy Simulation and Unsteady Reynolds-Averaged Navier-Stokes Simulations of Periodic Unsteady Flow in a Low-Pressure Turbine Cascade: A Comparison". Proceedings of the Institution of Mechanical Engineers, Part A-Journal of Power and Energy, 217, pp. 403-411.

[8] Medic, G., and Sharma, O. P., 2012. "Large-Eddy Simulation of Flow in a Low-Pressure Turbine Cascade". GT201268878, pp. 1239-1248.

[9] Langtry, R., and Menter, F., 2009. “Correlation-Based Transition Modeling for Unstructured Parallelized Computational Fluid Dynamics Codes". AIAA Journal, 47(12), pp. 2894-2906.

[10] Keadle, K., and McQuilling, M., 2013. "Evaluation of RANS Transition Modeling for High Lift LPT Flows at Low Reynolds Number". ASME Paper GT2013-95069.

[11] Walters, D. K. W., and Cokljat, D., 2008. "A ThreeEquation Eddy-Viscosity Model for Reynolds-Averaged Navier-Stokes Simulations of Transitional Flows". ASME Journal of Fluids Engineering, 130(12), p. 12401.

[12] Wu, X., Jacobs, R. G., Hunt, J. C. R., and Durbin, P. A., 1999. "Simulation of boundary layer transition induced by periodically passing wakes". Journal of Fluid Mechanics, 398, pp. 109-153.

[13] Michelassi, V., Fröhlich, J., Rodi, W., and Wissink, J. G., 2003. "Large-Eddy Simulation of Flow Around Low- 
Pressure Turbine Blade with Incoming Wakes". AIAA Journal, 41(11), pp. 2143-2156.

[14] Durbin, P., and Wu, X., 2007. "Transition Beneath Vortical Disturbances". Annual Review of Fluid Mechanics, 39, pp. 107-128.

[15] Edwards, T., and Sandberg, R. D., 2011. "CRAY Centre of Excellence Project report 2011". In www.hector.ac.uk/coe/pdf/HiPSTAR_OMP_Report.pdf.

[16] Sandberg, R. D., Pichler, R., and Chen, L. W., 2012. "Assessing the Sensitivity of Turbine Cascade Flow to Inflow Disturbances Using Direct Numerical Simulation". In 13th International Symposium for Unsteady Aerodynamics, Aeroacoustics and Aeroelasticity in Turbomachinery (ISUAAAT).

[17] Goldstein, D., Handler, R., and Sirovich, L., 1993. "Modeling a No-Slip Flow Boundary with an External Force Field". Journal of Computational Physics, 105, pp. 354366.

[18] Jeong, J., and Hussain, F., 1995. "On the Identification of a Vortex". Journal of Fluid Mechanics, 285, pp. 69-94.

[19] Denton, J. D., 1993. "Loss Mechanisms in Turbomachines". Journal of Turbomachinery, 115, pp. 621-656.

[20] Stewart, W. L., 1955. "Analysis of Two Dimensional Compressible Flow Loss Characteristics Downstream of Turbomachine Blade Rows in Terms of Basic Boundary Layer Characteristics". NACA TN 3515.

[21] Wilcox, D. C., 1998. Turbulence Modeling for CFD, 2nd ed. DCW Industries. 